\title{
HSP90 expression and its association with wighteone metabolite response in HER2-positive breast cancer cells
}

\author{
ZHONG-WEI CAO $^{1}$, QIAN ZENG ${ }^{2}$, HAI-JIANG PEI ${ }^{1}$, LI-DONG REN $^{1}$, HAI-ZHEN BAI ${ }^{1}$ and RI-NA NA ${ }^{1}$ \\ Departments of ${ }^{1}$ Thyroid, Breast, Hernia and Vascular Surgery and ${ }^{2}$ Neurology, \\ The Inner Mongolia Autonomous Region People's Hospital, Hohhot, Inner Mongolia Autonomous Region 010017, P.R. China
}

Received December 14, 2014; Accepted January 28, 2016

DOI: $10.3892 / \mathrm{ol} .2016 .4488$

\begin{abstract}
It is well known that heat shock protein 90 (HSP90) overexpression is correlated with poor prognosis and chemo-resistance in human malignant cancers. At the same time, wighteone, or 6-prenyl-5,7,4'-trihydroxyisoflavone, a major isoflavone component of the ornamental tall tree Erythrina suberosa, has been demonstrated to exhibit a potent anti-proliferative effect on human leukemia HL-60 cancer cell lines. In this study, the effects of wighteone on the proliferation of HER2-positive breast cancer cells were investigated, and the action mechanism was explored. MCF-7 HER2-positive breast cancer cells were treated with various concentrations of wighteone. The growth inhibitory rate of the cells was calculated by MTT assay, apoptosis was detected by flow cytometry, and the expression level of HSP90 was assessed by western blot analysis. The addition of wighteone at concentrations ranging from $1-10 \mathrm{~g} / \mathrm{ml}$ in the medium for $48 \mathrm{~h}$ had a marked inhibition on the proliferation of HER2-positive cancer cell lines. The growth inhibitory rates with $0.5,2$ or $8 \mathrm{mM}$ wighteone were significantly higher compared with the control group. Apoptosis in the wighteone-treated cells was also significantly higher compared with the control group. The expression level of HSP90 in the wighteone group was significantly lower than that in the control group. Our findings demonstrated that wighteone effectively inhibited the proliferation of HER2-positive cancer cell lines, and this is considered to be the result of downregulating HSP90 receptor and downstream signaling.
\end{abstract}

\section{Introduction}

Breast cancer is one of the most common and significant diseases affecting females. Breast tumors are comprised of phenotypically diverse populations of breast cancer cells. Current estimates

Correspondence to: Dr Zhong-Wei Cao, Department of Thyroid, Breast, Hernia and Vascular Surgery, The Inner Mongolia Autonomous Region People's Hospital, 20 Zhaowuda Road, Hohhot, Inner Mongolia Autonomous Region 010017, P.R. China

E-mail: caozhongwei85@gmail.com

Key words: wighteone, metabolite, breast cancer, protein expression, apoptosis indicate that one in eight females in American who reach the age of 95 is likely to develop breast cancer (1). The treatment of advanced breast cancer is often unsuccessful and may cause disfigurement, making early detection a high priority in the medical management of the disease. The level of morbidity and high incidence of this disease is notable. Within the next two decades the incidence of cancer is expected to rise worldwide. It is noted that the management of cancer is still not under control and new effective drugs are lacking (2). Proteins associated with the human epidermal growth-factor receptor kinase (ERBB or HER) signaling network have proven to be useful targets for diagnostic imaging with radioimmunoconjugates due to their overexpression in various cancer phenotypes. In particular, the overexpression of the HER2/neu (also known as ERBB2) was revealed to be associated with increased tumor aggression, metastatic potential and poor prognosis for disease-free survival in patients with breast, colorectal, ovarian, lung, prostate and salivary gland tumors $(3,4)$.

HER2/neu was revealed to be a key target for anticancer drugs due to its intrinsic involvement in the phosphatidylinositol-3-kinase-Akt/protein kinase B and the mitogen-activated protein kinase (MAPK) pathways. These pathways suppress apoptosis and promote tumor cell survival, gene transcription, angiogenesis, cellular proliferation, migration, mitosis and differentiation. There are three notable types of anti-HER $2 / \mathrm{neu}$ therapeutics; namely, monoclonal antibodies (mAbs) directed against extracellular ligand-binding and dimerization epitopes, tyrosine-kinase (TK) inhibitors and heat shock protein 90 (HSP90) inhibitors. Examples of each therapeutic type include pertuzumab and trastuzumab (which block dimerization and suppress signaling by binding to extracellular domains II and IV, respectively), the HER2/neu TK inhibitor lapatinib, and HSP90 inhibitors including geldanamycin derivatives, SNX-5422, NVP-AUY922, BIIB021 and PU-H71 (5-10). HSP90 is a molecular chaperone protein essential for the function of the multiple growth and survival pathways required for the maintenance and progression of cancer (11). In breast cancer in particular, the overexpression of HSP90 is associated with poor outcome and decreased survival $(12,13)$. Trastuzumab and related $\mathrm{mAb}$ fragments have been radiolabeled with a wide range of radionuclides, and quantitative immune positron emission tomography (PET) imaging has been employed to assess the effect of Hsp90 inhibitors on the expression levels of HER2/neu (14-18). The quantification of changes in HER2/neu 
expression in response to HSP90 treatment has the potential to facilitate patient-specific dose regimes.

During the last few years, the focus of drug development has shifted to natural chemotherapeutic agents from plants, which may be further modified to enhance their potential and reduce their side effects. Natural products have been particularly useful in cancer treatment, and if biologicals and vaccines are discounted, then $75 \%$ of approved chemotherapeutic agents are natural agents (19). Erythrina is a genus of flowering plants in the pea family, Fabaceae, with 130 species reported. It is distributed worldwide in tropical and subtropical regions in addition to certain more temperate regions, and $\sim 50 \%$ of its varieties have been studied. The alkaloids isolated from various species of Erythrina demonstrate hypotensive, anticonvulsant, hypnotic and analgesic properties, among others (20). Erythrina plant species are widely used in folk medicine to treat health conditions including agitation, insomnia, anxiety and inflammation $(21,22)$. A previous study has revealed that an alcoholic extract of the stem bark of $E$. suberosa induces apoptosis in human promyelocytic leukemia HL-60 cells (23). The aim of the current study was to investigate the anticancer potential of the flavonoid wighteone isolated from the stem bark of E. suberosa, and to study HSP90 expression and its correlation with wighteone metabolite response in HER2-positive breast cancer cells.

\section{Materials and methods}

Reagents and cell lines. The breast cancer cell line MCF-7 was provided by the Breast Cancer Research Department of the Inner Mongolia Autonomous Region People's Hospital, Hohhot, China. Cells were cultured in Dulbecco's modified Eagle's medium (DMEM) with $10 \%$ fetal bovine serum (FBS) $\left(\right.$ Gibco $^{\circledR}$; Thermo Fisher Scientific, Inc., Waltham, MA, USA), $1 \%$ glutamine (Sigma-Aldrich, St. Louis, MO, USA), $100 \mathrm{IU} / \mathrm{ml}$ penicillin (Sigma-Aldrich) and $100 \mathrm{mg} / \mathrm{l} \mathrm{strep-}$ tomycin (Sigma-Aldrich). The MTT kit was purchased from Sigma-Aldrich. Annexin V-fluorescein isothiocyanate (FITC) and the propidium iodide (PI) Annexin V-EGFP apoptosis detection kit were purchased from KGI Chemical Corporations (Nanjing, China), monoclonal mouse anti-rat HSP90 antibody (cat. no. ab13492; 1:1,000) from Abcam (Cambridge, UK) and monoclonal mouse anti-rat GAPDH antibody (cat. no. sc-32233; 1:1,000) from Santa Cruz Biotechnology, Inc. (Dallas, TX, USA). Wighteone was obtained as in previous studies (2).

3-(4,5-dimethylthiazolyl)-2,5-iphenyltetrazolium bromide (MTT) assay. Cell viability was determined using the MTT assay (Sigma-Aldrich). Firstly, cells were divided into four groups: group A: cells were treated with phosphate-buffered saline (PBS); group B: cells were treated with $0.5 \mathrm{mM} / 1$ wighteone; group $\mathrm{C}$ : cells were treated with $5.0 \mathrm{mM} / \mathrm{l}$ wighteone; group D: cells were treated with $10.0 \mathrm{mM} / 1$ wighteone. Cells from groups A, B, C and D were plated in 96-well plates ( $2 \times 10^{4}$ cells/well) and cultured overnight in DMEM with $10 \%$ FBS. Following culture for 12, 24 and $48 \mathrm{~h}$, the cells were treated with $0.5 \mathrm{mg} / \mathrm{ml} \mathrm{MTT}$ for $4 \mathrm{~h}$ and lysed with dimethyl sulfoxide (Sigma-Aldrich). Absorbance rates were measured at $560 \mathrm{~nm}$ using a microplate reader (iMark ${ }^{\mathrm{TM}}$ Microplate Absorbance Reader; Bio-Rad Laboratories, Inc., Hercules, CA, USA).

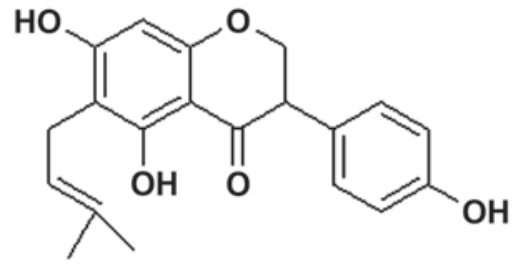

Figure 1. Structure of wighteone.

Annexin V-FITC/PI analysis. Apoptosis detection was conducted using the Annexin V-FITC/PI apoptosis detection kit, according to the manufacturer's instructions. Briefly, all four groups of cells were harvested by trypsinization, washed in PBS and stained with Annexin V-FITC conjugate and PI. Cells were then analyzed by flow cytometry (BD FACSCalibur $^{\mathrm{TM}}$, BD Biosciences, San Jose, NJ, USA) using BD CellQuest acquisition and analysis software.

Western blot analysis. The total volume of all formulations was $10 \mu \mathrm{l}$. The cells were diverted into a $1.5 \mathrm{ml}$ Eppendorf tube (Eppendorf, Hamburg, Germany) which contained radioimmunoprecipitation assay buffer schizolysis liquid with protease inhibitor (Sigma-Aldrich). After being put on ice for 5-10 min, the mixture was agitated to make it fully dissolve, and put on ice for $30 \mathrm{~min}$. Then the mixture was centrifuged $(100 \mathrm{x} \mathrm{g})$ at $4^{\circ} \mathrm{C}$ for $20 \mathrm{~min}$, and the top clear liquid was collected and electrophoresed.

Statistical analysis. The data are presented as the mean \pm standard deviation from at least three independent experiments. One way analysis of variance was used and all data analyses were performed using SPSS 13.0 software (SPSS, Inc., Chicago, IL, USA). $\mathrm{P}<0.05$ was considered to indicate a statistically significance difference.

\section{Results}

Wighteone inhibits MCF-7 cell proliferation. The structure of wighteone is shown in Fig. 1. The results obtained from the MTT assay revealed that treatment with wighteone significantly inhibited cancer cell proliferation compared with the control PBS group $(\mathrm{P}=0.03)$. The results are shown in Table I and Figs. 2 and 3, Moreover, when increasing the concentrations of wighteone, the proliferation rates were gradually decreased.

Apoptosis. The experimental studies revealed that the apoptosis rates in groups A, B, C and D were 3.02, 4.22, 6.54 and $14.98 \%$, respectively. However, only the apoptotic rate in group D differed significantly compared with that in group A $(\mathrm{P}=0.003)$. The apoptotic rate in group $\mathrm{D}$ also demonstrated a significant difference compared with that in group $\mathrm{B}$ ( $\mathrm{P}=0.008$; Fig. 4).

HSP90 protein expression. HSP90 protein expression of MCF-7 cells following treatment with wighteone is shown in Fig. 5. HSP90 expression was significantly lower $(\mathrm{P}=0.03)$ in groups B, C and D compared with that in group A. Moreover, the HSP90 expression at $48 \mathrm{~h}$ was significantly lower compared with that at $24 \mathrm{~h}(\mathrm{P}=0.04)$. 
Table I. Inhibition of MCF-7 cell proliferation following treatment with wighteone.

Inhibition rate of $\mathrm{MCF}-7$ cancer cells (mean $\pm \mathrm{SD}$ )

\begin{tabular}{lccrr} 
& & \multicolumn{2}{c}{$24 \mathrm{~h}$} & \\
\hline Group & Wighteone, $\mathrm{mM} / \mathrm{l}$ & $12 \mathrm{~h}$ & 0 & 0 \\
A & Control & 0 & $5.62 \pm 0.12$ & $6.44 \pm 0.07$ \\
B & 0.5 & $4.90 \pm 0.27$ & $15.18 \pm 0.28$ & $21.36 \pm 0.12$ \\
C & 2.0 & $6.34 \pm 0.24$ & $21.16 \pm 0.11$ & $29.17 \pm 0.02$ \\
D & 8.0 & $7.73 \pm 0.33$ & & \\
\hline
\end{tabular}
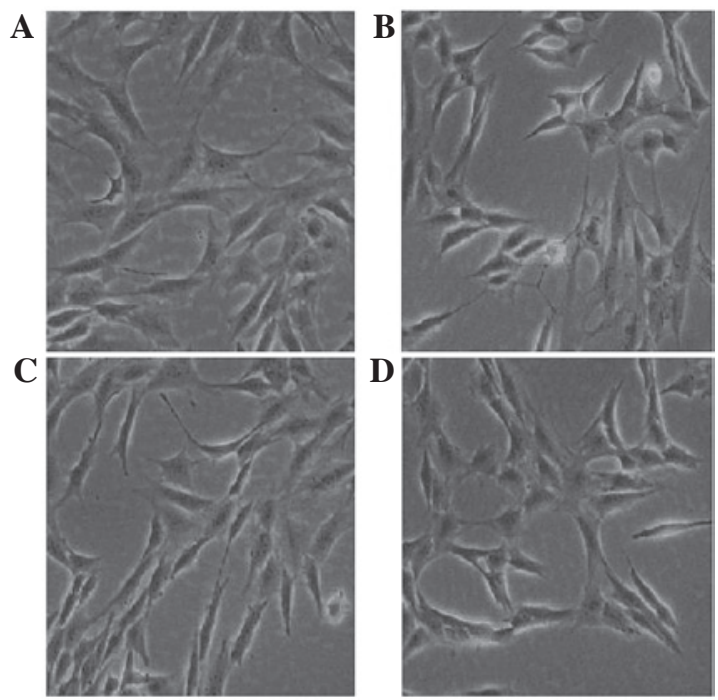

Figure 2. Inhibition of cell proliferation following treatment with various concentrations of wighteone at $48 \mathrm{~h}$. (A) Phosphate-buffered saline; (B) $0.5 \mathrm{mM}$ wighteone; (C) $2 \mathrm{mM}$ wighteone; (D) $8 \mathrm{mM}$ wighteone. Cells were visualized by phase contrast and fluorescence microscopy at a magnification of x100.

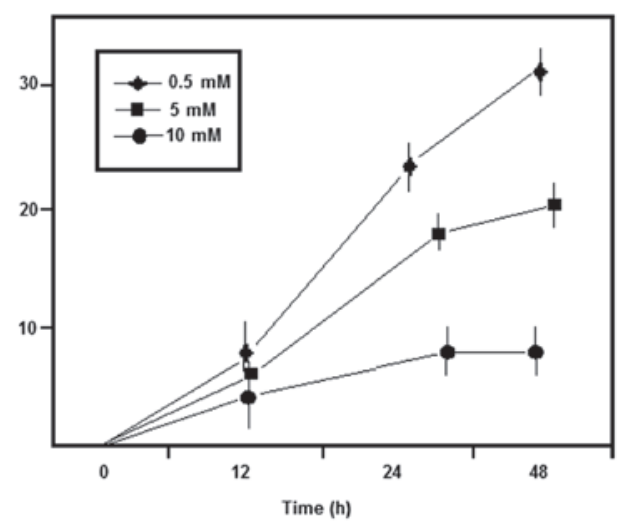

Figure 3. Inhibition of cell proliferation by wighteone in vitro. There was a statistically significant difference in the inhibition rate with $0.5,5$ and $10 \mathrm{mM}$ wighteone vs. the control group $(\mathrm{P}<0.05)$. Furthermore, with the increasing wighteone concentration the proliferation rates gradually decreased $(\mathrm{P}<0.05)$.

\section{Discussion}

The use of plants as medicine dates back thousands of years, and they have become an essential source of the mainstream pharmacopoeia. Nature is an attractive source of new therapeutic candidate compounds as a vast chemical diversity
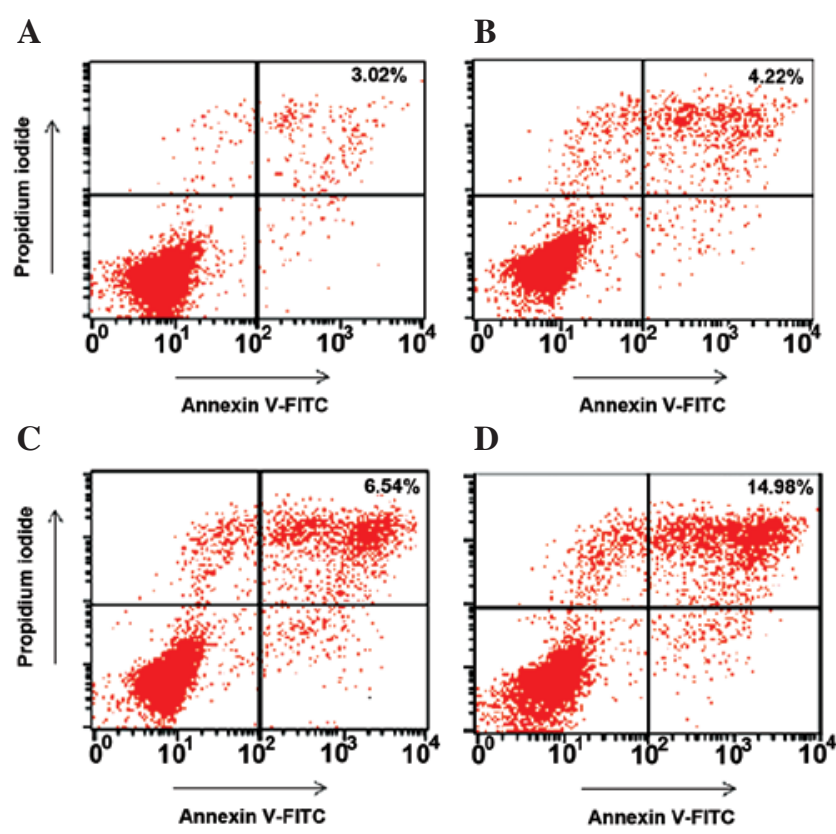

Figure 4. Wighteone-induced breast cancer cell apoptosis. The apoptotic rate in groups A, B, C and D was 3.02, 4.22, 6.54 and $14.98 \%$, respectively. The apoptotic rate in group D differed significantly from that in group A $(\mathrm{P}=0.003)$. The apoptotic rate in group $\mathrm{D}$ also demonstrated a significant difference to that in group $B(P=0.008)$. (A) Control group; (B) $0.5 \mathrm{mM}$ group; (C) $5 \mathrm{mM}$ group; (D) $10 \mathrm{mM}$ group.

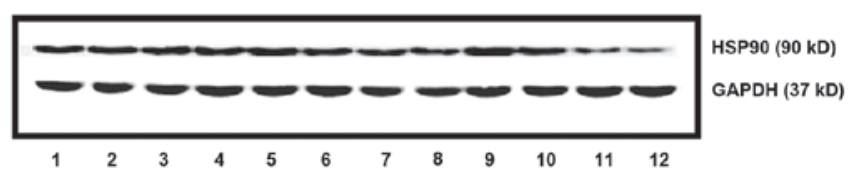

Figure 5. Heat shock protein 90 (HSP90) protein expression was assessed by western blot analysis at 0,24 and $48 \mathrm{~h}$. Channels 1,5 and 9 correspond to group A; channels 2, 6 and 10 to group B; channels 3,7 and 11 to group C; and channels 4,8 and 12 to group D. HSP90 expression was significantly lower in groups B, C and D compared with group A. In addition, HSP90 expression at $72 \mathrm{~h}$ was significantly lower $(\mathrm{P}<0.05)$ compared with that at $36 \mathrm{~h}$.

has been observed in diverse plant species, animals, marine organisms and microorganisms. The advantage of botanical compounds is their ability to support the treatment of all phases of cancer. These compounds regularly interact with several targets simultaneously and often act synergistically. The Erythrina plant species is widely used in folk medicine to treat various health problems including agitation, insomnia, and anxiolytic and inflammatory processes. Recently, the 
focus of drug development has shifted to the natural chemotherapeutic agents from plants, which may further be modified to enhance their potential and reduce their side effects. The identification of drugs from medicinal plants has played a significant role in the treatment of cancer; in fact, the majority of new clinical applications of plant secondary metabolites and derivatives over the last half century have been towards combating cancer. This forms the basis of the present study, in which our crucial aim was to develop a holistic molecular approach in consideration of the fact that several genes are mutated in cancer cells, which protects them from self-demise.

The predictive role of HSP90 tumor expression as a biomarker of activity of specific inhibitors remains unclear, although its overexpression is considered to confer a poor prognostic outcome in various tumors, including lung cancer, breast cancer and leukemia $(24,25)$. With particular regard to breast cancer, the independent poor prognostic role of HSP90 overexpression in multivariate modeling was previously demonstrated in a large series of over 600 patients with a follow-up period of more than 10 years, together with large tumor size, nodal positivity, lower progestin receptor level and high HER2 level (26).

All the experimental results revealed that with increasing concentrations of wighteone the inhibition of proliferation of MCF-7 cells gradually increased. The apoptotic rate in the wighteone-treated groups was higher compared with the control group $(\mathrm{P}<0.05)$. From this, it is clear that wighteone induces MCF-7 cell apoptosis in vitro and restrains cell proliferation. The activation of the AKT and MAPK pathways and the high expression of HSP90 protein are common in HER2-positive breast cancer (27) and there is a positive correlation between HSP90 and breast cancer pathological stage, local recurrence and distant metastasis, all of which result in poor patient prognosis (28). Our study reveals that wighteone blocks the expression of HSP90 protein in MCF-7 cells, and that the inhibitory effect is enhanced with increasing drug concentrations. Wighteone is able to control proliferation and promote apoptosis in cancer cells, and it is possible that wighteone decreases the expression of HSP90 protein, which downregulates the key downstream molecular activation of the AKT and MAPK pathways.

In conclusion, the present study confirmed that wighteone significantly inhibits proliferation and promotes apoptosis in HER2-positive breast cancer cells, and that this may be associated with the inhibition of HSP90 protein expression in cancer cells.

\section{Acknowledgements}

This study was supported by The Inner Mongolia Autonomous Region People's Hospital (grant no. 20110936) and the Natural Science Foundation of Inner Mongolia (grant no. 2014MS0801). The authors kindly acknowledge and thank these institutions for their support throughout this study.

\section{References}

1. American Cancer Society: Cancer Facts \& Figures 1994. American Cancer Society, Atlanta, GA, pp13, 1994.
2. Kumar S, Pathania AS, Saxena AK, et al: The anticancer potential of flavonoids isolated from the stem bark of Erythrina suberosa through induction of apoptosis and inhibition of STAT signaling pathway in human leukemia HL-60 cells. Chem Biol Interact 205: 128-137, 2013.

3. Hanahan D and Weinberg RA: The hallmarks of cancer. Cell 100: 57-70, 2000.

4. Baselga $J$ and Swain SM: Novel anticancer targets: revisiting ERBB2 and discovering ERBB3. Nat Rev Cancer 9: 463-475, 2009.

5. Beliakoff J and Whitesell L: Hsp90: an emerging target for breast cancer therapy. Anticancer Drugs 15: 651-662, 2004.

6. Mendelsohn J and Baselga J: Epidermal growth factor receptor targeting in cancer. Semin Oncol 33: 369-385, 2006.

7. Solit DB and Chiosis G: Development and application of Hsp90 inhibitors. Drug Discov Today 13: 38-43, 2008.

8. Mendelsohn J and Baselga J: Status of epidermal growth factor receptor antagonists in the biology and treatment of cancer. J Clin Oncol 21: 2787-2799, 2003.

9. Citri A, Kochupurakkal BS and Yarden Y: The Achilles heel of ErbB-2/HER2: regulation by the Hsp90 chaperone machine and potential for pharmacological intervention. Cell Cycle 3:51-60, 2004.

10. Taldone T, Sun W and Chiosis G: Discovery and development of heat shock protein 90 inhibitors. Bioorg Med Chem 17: 2225-2235, 2009.

11. Whitesell L and Lindquist SL: HSP90 and the chaperoning of cancer. Nat Rev Cancer 5: 761-772, 2005.

12. Pick E, Kluger Y, Giltnane JM, et al: High HSP90 expression is associated with decreased survival in breast cancer. Cancer Res 67: 2932-2937, 2007.

13. Song CH, Park SY, Eom KY, et al: Potential prognostic value of heat-shock protein 90 in the presence of phosphatidylinositol-3-kinase overexpression or loss of PTEN, in invasive breast cancers. Breast Cancer Res 12: R20, 2010.

14. Smith-Jones PM, Solit D, Afroze F, Rosen N and Larson SM: Early tumor response to Hsp90 therapy using HER2 PET: comparison with 18F-FDG PET. J Nucl Med 47: 793-796, 2006.

15. CostantiniDL,BatemanK,McLartyK,etal:Trastuzumab-resistant breast cancer cells remain sensitive to the Auger electron-emitting radiotherapeutic agent $111 \mathrm{In}-\mathrm{NLS}$-trastuzumab and are radiosensitized by methotrexate. J Nucl Med 49: 1498-1505, 2008.

16. Orlova A, Wållberg $\mathrm{H}$, Stone-Elander $\mathrm{S}$ and Tolmachev V: On the selection of a tracer for PET imaging of HER2-expressing tumors: direct comparison of a 124I-labeled affibody molecule and trastuzumab in murine xenograft model. J Nucl Med 50: 417-425, 2009.

17. Kramer-Marek G, Kiesewetter DO and Capala J: Changes in HER 2 expression in breast cancer xenografts after therapy can be quantified using PET and (18)F-labeled affibody molecules. J Nucl Med 50: 1131-1139, 2009.

18. Dijkers EC, Kosterink JG, Rademaker AP, et al: Development and characterization of clinical-grade 89Zr-trastuzumab for HER $2 /$ neu immunoPET imaging. J Nucl Med 50: 974-981, 2009.

19. Newman DJ and Cragg GM: Natural products as sources of new drugs over the 30 years from 1981 to 2010. J Nat Prod 75: 311-335, 2012.

20. Hargreaves RT, Jonson RD, Millington DS, et al: Alkaloids of American species of Erythrina. Lloydia 37: 569-580, 1974.

21. Chopra RN, Nayar SL and Chopra IC (eds): Glossary of Indian Medicinal Plants. CSIR, New Delhi, 1956.

22. Garín-Aguilar ME, Luna JE, Soto-Hernández M, et al: Effect of crude extracts of Erythrina americana Mill. on aggressive behavior in rats. J Ethnopharmacol 69: 189-196, 2000.

23. Agrawal SK, Agrawal M, Sharma PR, et al: Induction of apoptosis in human promyelocytic leukemia HL60 cells by an extract from Erythrina suberosa stem bark. Nutr Cancer 63: 802-813, 2011.

24. Gallegos Ruiz MI, Floor K, Roepman P, Rodriguez JA, et al: Integration of gene dosage and gene expression in non-small cell lung cancer, identification of HSP90 as potential target. PLoS One 3: e0001722, 2008.

25. Garcia-Carbonero R, Carnero A and Paz-Ares L: Inhibition of HSP90 molecular chaperones: moving into the clinic. Lancet Oncol 14: e358-e369, 2013.

26. Pick E, Kluger Y, Giltnane JM, Moeder C, Camp RL, Rimm DL and Kluger HM: High HSP90 expression is associated with decreased survival in breast cancer. Cancer Res 67: 2932-2937, 2007.

27. Ciocca DR, Clark GM, Tandon AK, Fuqua SA, Welch WJ and McGuire WL: Heat shock protein hsp70 in patients with axillary lymph node-negative breast cancer: prognostic implications. J Natl Cancer Inst 85: 570-574, 1993.

28. Kim LS and Kim JH: Heat shock protein as molecular target for breast cancer therapeutics. J Breast Cancer 14: 167-174, 2011. 